\title{
Z Research Square \\ Identification of Enhancer RNA CDK6-AS1 as a Potential Novel Prognostic Biomarker in Gastric Cancer
}

\author{
Shifeng Yang \\ The Second Affiliated Hospital of Harbin Medical University \\ Xiaoming Zou (D zou4930@163.com ) \\ The Second Affiliated Hospital of Harbin Medical University \\ Hao Yang \\ The Second Affiliated Hospital of Harbin Medical University \\ Jiacheng Li \\ The Second Affiliated Hospital of Harbin Medical University \\ Ange Zhang \\ The Second Affiliated Hospital of Harbin Medical University \\ Llsha Zhang \\ The Second Affiliated Hospital of Harbin Medical University \\ Changjian Li \\ The Second Affiliated Hospital of Harbin Medical University \\ Lei Zhu \\ The Second Affiliated Hospital of Harbin Medical University \\ Zhen Ma \\ The Second Affiliated Hospital of Harbin Medical University
}

\section{Research Article}

Keywords: CDK6-AS1, CDK6,enhancer RNA, gastric cancer, prognostic biomarker

Posted Date: December 16th, 2021

DOI: https://doi.org/10.21203/rs.3.rs-1159260/v1

License: @ (1) This work is licensed under a Creative Commons Attribution 4.0 International License. Read Full License 


\section{Abstract}

Background.

The aim of this study was to confirm enhancer RNAs (eRNAs) in gastric cancer and its clincial utility.

\section{Methods.}

We used cox survival analysis and relevance analysis to identify the candidate eRNAs in gastric cancer. Moreover, we performed GO and Reactome pathway enrichment to found the potential functions of eRNAs.Correlation between eRNA, tumor-infiltrating immune cells and drug sensitivity was then analyzed.

\section{Results.}

CDK6-AS1 may serve as a poor independent prognostic biomarker candidate in gastric cancer with positive correlation with its target gene CDK6. Low CDK6AS1 expression group showed more frequent mutated driver genes than high expression ones. Moreover, CDK6-AS1 is involved in key oncogenic pathway as cell cycle and RNA transcription. CDK6-AS1 also shows dysregulations and associations with prognosis at pan-cancer level. This eRNA may also associated with immune cell infiltration and drug sensitivity.

\section{Conclusion:}

CDK6-AS1 may be a potential prognostic biomarker for gastric cancer, predict chemotherapeutic drugs sensitivity of gastric cancer.

\section{Background}

Gastric cancer was the world's fifth-most commonly diagnosed cancer type and sixth cause of cancer mortality in 2018, responsible for 1,033,701 newly diagnosed cases and 782,685 deaths worldwide(1). During the past decades, gastric cancer maintains a high case fatality rate of $75 \%$ throughout most of the world and is a main contributor to global disability-adjusted life-year burden(, 3). Recently, The prognosis of gastric cancer had improvement and the treatment technology has been significantly improved(4 $₫ 5)$.

ThepastdecadeshavewitnessedtherapidprogressofknowledgeabouttherolenoncodingRNAsplayedinawiderangeofcancers(6).Moreandmoreresearchespaidatten RNAs can activate enhancer activity independently, and can also cooperate with other transcription factors to initiate the formation of the enhancer promoter loop, thereby activating the expression of downstream genes and pathways $(9,10)$. Dysregulation of enhancer RNAs could result in a wide range of human cancers, specifically in oncogenic signaling pathway(11). For instance, Kallikrein-elated peptidase 3 eRNA in prostate cancer was found to promote the transcription of downstream androgen receptor gene and propel cancer cell proliferation(12). Recent study have also shown some dysregulated eRNAs could serve as prognostic biomarkers in a range of cancers such as head and neck squamous cell carcinoma, lung and colon adenocarcinoma(13-15). Nevertheless, the predictive factors of gastric cancer didn't found, as well as their underlying functions with tumor microenvironment (TME) cells and chemosensitivity.

In this study, we identified potential prognostic eRNA, CDK6-AS1, and its target gene in gastric cancer. Pathway enrichment analyses were performed to explore the potential function CDK6-AS1 may played during tumorigenesis. Further, we validated CDK6-AS1 expression and overall survival at pan-cancer level. We also found that CDK6-AS1 is associated with T cell infiltration and therapy sensitivity.

\section{Materials And Methods}

\section{Data collection and processing}

The information of 33 dataset were downloaded from the UCSC Xena The Cancer Genome Atlas (TCGA) hub (https://xena.ucsc.edu) (16). The dataset included 407 gastric cancer tissues with 32 normal tissues, and 9951 other tumors from different types. The RNA expression matrix were transformed to $\log 2($ FPKM+1). Ensemble transcript IDs were con-verted to their corresponding GENCODE v19 using GTF annotation files from human. Enhancer RNAs and their target genes information was obtained from putative literature, which were previously identified by the PreSTIGE method(17).

\section{Identification of predictive eRNAs in gastric cancer}

To avoid bias, patients with survival time less than one month were filtered out. Finally, 375 patients passed quality control and were used in the following analysis (Table 1). Then, The survival-associated eRNAs were screened using Cox regression model, with age, gender, and tumor stage adjusted as covariates. We set $\mathrm{P}<0.05$ as cut-off values.

\section{Analysis of significantly mutated genes}

The R package maftools was used to compare the mutant frequencies of significantly mutated genes between CDK6-AS1 high and low expression group. Mutant types including frame shit deletion, splice site, frame shift insertion, missense mutation, nonsense mutation, multiple hist, in frame deletion were taken into analysis. 


\section{Gene enrichment analysis}

Gene Ontology (GO) functional analysis was performed using ClusterProfiler package in R software, so as for Reactome pathway analysis of eRNArelated coding genes based on co-expression analysis. Specifically, the GO analysis revealed the function in biology process(BP), cell component(CC), and molecular function(MF).

To avoid accumulation of type- $₫$ errors, enrichment items meet the false discovery rate $(F D R)<0.05$ were considered as significant.

\section{Validation in TCGA pan-cancer cohort}

The expression data of CDK6-AS1 and its target gene CDK6 at pan-cancer level were obtained as described above. Patients were classified into low- and highexpression groups according to the median value of the expression of CDK6-AS1, then the Cox regression method compare the overall survival difference between the two groups. Covariates gender, age, and tumor stage were adjust in the cox model. Spearman's coefficient applied to correlation analysis.

\section{Analysis of Immune Cell Infiltrates}

To evaluate the relationship between tumor-infiltrating immune (TIL) cells with the expression of CDK6-AS1 in gastric cancer, we first estimated the expressed fraction of TIL cells with SSGSEA algorithm by comparing the gastric cancer gene expression matrix with those of the signatures from 9 reported TIL cell types(18). Then, the relationship of proportion matrix for the 9 TIL cells with CDK6-AS1 were calculated by the Spearman's correlation analysis.

\section{Prediction of chemosensitivity}

The R package pRRophetic(19), based on the pharmacogenomics database of Cancer Genome Project (CGP) cell line data and the Cancer Cell Line Encyclopedia (CCLE), was utilized to predict chemotherapeutic sensitivity for breast cancer patients by estimation of IC50 (half maximal inhibitory concentration). Default settings were used for prediction model, including "stomach cancer" for reference tissue type and "cvFold=10" for ridge regression model training.

\section{Statistical analysis}

R software (Version 3.6.2) applied to analysis this study. The statistical results are expressed by mean \pm standard deviation (M \pm SD), the data comparison of two groups was used to Wilcoxon rank-sum test, $P<0.05$ was the difference with statistical significance.

\section{Results}

\section{Screening the key eRNA in gastric cancer}

23 eRNAs were identified. Eight eRNA-target pair met the criteria ( Spearman $r \geq 0.3$ and $F D R<0.05$ ) and were selected (Table S1). Of these, CDK6-AS1 exhibited the lowest Cox model $P$ value, and was therefore considered to be the candidate markers. Patients in the CDK6-AS1 high-expression group survived shorter than that in low-expression group (3-year OS: HR=1.68, $P=3.84 \times 10-3$; 5-year OS: HR=1.62, $P=5.64 \times 10-3$, Fig1 A-B). In addition, CDK6-AS1 shown higher expression in unpaired and paired tumor tissues than that in normal tissues (Unpaired: $P=8.00 \times 10-3$, paired: $P=0.046$, Fig1C-D). We also observed positive correlation between CDK6-AS1 and its target gene CDK6 (Spearman $r=0.38, P=1.68 \times 10-14$ ). We further investigated the connections between the clinical features of gastric patients and CDK6-AS1 expression. We found that CDK6-AS1 expressed higher in patients below 60 years $(P=0.022$, Fig2A). And CDK6-AS1 was significantly linked to clinical stage ( $\nabla$ v.s. $\otimes, P=0.048$, Fig2C $)$. Other clinical characteristics were found no clear correlation with CDK6-AS1 ( $P>0.05$, Fig2B,D-H). As driver genes mutation were crucial to tumor growth, we also compare the frequencies of significantly mutated genes between high and low CDK6-AS1 expression groups. We found that several classic gastric cancer driver genes were more frequent mutated in low CDK6-AS1 expression group compared to that in high group, such as ARID1A and PIK3CA (Fig3 A-B, Table S2).

\section{Pathway enrichment analysis of CDK6-AS1 co-expressed genes}

To further explore the function and related pathways CDK6-AS1 involved in gastric cancer, we performed co-expression analysis between CDK6-AS1

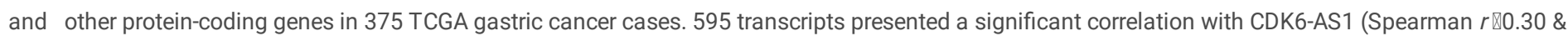
$F D R<0.05$ ). GO and Reactome enrichment analysis were performed. Results from TOP10 GO pathways in biological process (BP), molecular function (MF), and cellular component (CC) are shown in Fig4A, Table S3. In BP, the terms were mainly related to RNA transportation. In MF, the terms were related to exoribonuclease activity; In CC, terms were involved in nuclear chromosomal region. Enrichment from Reactome pathway database indicated that CDK6-AS1 related co-expressed genes were mainly in cell cycle and mitosis (Fig4B, Table S5), key signals to tumor cell proliferation. The cell cycle pathway-related genes with a Spearman's correlation $r>0.3$ are showed in Table S5. Taken together, CDK6-AS1 and its related genes may involve in gene transcription and cell cycle process, which are essential to malignant progression. 
To determine the CDK6-AS1 expression, prognosis and the correlation with its target gene at pan-cancer level, we analyze in 33 tumor cohorts in TCGA database. CDK6-AS1 shown more expression in tumor tissues than that in adjacent-normal tissues in 16 tumor types: COAD, DLBC, ESCA, GBM, HNSC, KIRP, LAML, LIHC, LUSC, OV, PAAD, PCPG, READ, SARC, THYM, and UCS. Meanwhile, 6 types shown more expression in normal tissues than malignant ones: ACC, BRCA, KICH, PCPG, and TGCT (Fig 5A). In terms of survival analysis, high expressed CDK6-AS1

group was related to poor prognosis in BLCA, HNSC, KIRC, LGG, LUAD, MESO, and THCA (Fig5 B-H), while shown better prognosis in UVM (Fig 5 I). Further analysis was performed concerning the correlation of CDK6-AS1 expression with its target gene CDK6. We found that CDK6-

AS1 shown correlation with its target in 29 types of tumor (Table S6). Taken together, CDK6-AS1 is dysregulated and could influence prognosis around a range of different cancer types.Representative immunohistochemical staining for gastric cance tumor-infiltrating target gene CDK6. Scale bar, 50 mm(Fig $5 \mathrm{~J})$.Western blot results showed that CDK6 was significantly expressed in metastatic gastric cancer cells MKN-45 and in-situ gastric cancer cells $\mathrm{HGC}-27$, while GES- 1 was not significantly expressed in normal gastric mucosa epithelial cells (Fig 5K).

\section{Correlation between CDK6-AS1 and tumor-infiltrating immune cells}

Since tumor-infiltrating immune cells are important players in tumor microenvironment and have been reported to influence the survival of gastric cancer patients, we therefore analyzed the association between CDK6-AS1 expression and infiltrating immune cells proportion. Higher CDK6-AS1 expression shown negative tendencies with almost all inferred immune cells enrichment scores (Fig6A-l), and shown significance among Thelper (Spearman $r=-0.20, P<0.001$, Fig6C), Treg (Spearman $r=-0.10, P=0.044$, Fig6D), and neutrophils cell clusters (Spearman $r=-0$. 14, $P=0.008$, Fig6l). Taken together, it seems that high CDK6-AS1 expression may hamper immune microenvironment cells infiltration, both innate and adaptive cell clusters, which may have effect on anti-tumor immunity.

\section{Drug sensitivity prediction}

Since chemosensitivity or resistance is related with gastric cancer clinical prognosis, we then explored the chemosensitivity of high and low CDK6-AS1 expression groups. Ridge regression model was used to predict individual drug sensitivities. One commonly used chemotherapy drugs, in gastric cancer therapy, cisplatin, showed more sensitivities in high CDK6-AS1 expression group compared to that in low expression ones ( $P=0.024$, Fig7A). Conversely, drug paclitaxel showed more sensitivity in low expression group $(P=0.040)$. We also explore the expression of immunotherapy and target therapy markers. Immunotherapy marker, PD-L1, expressed higher in low CDK6-AS1 expression group, which may indicate more sensitivity to anti-PD-L1 therapy (Fig7B).

\section{Discussion}

In our research, we found that CDK6-AS1 may serve as a poor independent prognostic biomarker candidate in gastric cancer with positive correlation with its target gene CDK6. Low CDK6-AS1 expression group showed more frequent mutated driver genes than high expression ones. Moreover, CDK6-AS1 is involved in key oncogenic pathway as cell cycle and RNA transcription. CDK6-AS1 also shows dysregulations and associations with prognosis at pan-cancer level.

Some researches had demonstrated that Enhancer RNAs worked by regulating the target genes and forming chromatin loop(7,

20).AresearchshowedthateRNAofACTRT1 couldlowertheexpressionoftargetgenesandpromotethedevelopment ofcancer(21).

Inourstudy,weconfirmedthatCDK6-AS1 wasassociatedwithsurvivalineighttypesoftumors(BLCA,HNSC,KIRC,LGG,LUAD,MESO,THCAandUVM), moreover,CDK6AS1 expressionwascorrelatedwiththatofitstargetgene,CDK6,in 29tumortypes.AndwesuggestedthatCDK6-AS1actedasanindependentpredictor ofgastriccancer.

CDK6 (cyclin-dependent kinases 6), could form complexes D-type cyclins (D1, D2 and D3) and progress cells to early G1 phase (22). CDK4/6-cyclin D complexes is regulated by Cip/Kip proteins which had impacted on nuclear translocation of complexes(23, 24). Some researches had reported that overexpression of CDK6 could affect on lymphoma, leukemia and other malignancies. Although there didn't found the relation between mutations in CDK6 and diseases, CDK6 had served as hub genes in the acute myeloid leukemia $(A M L)(25,26)$. The cyclin D-CDK4/6 axis is commonly expression in breast cancer. The another effect of CDK4/6 inhibitors was to effect immunity of antitumor. Zhang et al suggested that CDK4 negatively regulates programmed cell death 1 ligand 1 (PD-L1) protein stability, moreover, CDK4 and PD-L1 levels negatively correlate with treatment of tumor(27,28).

Since tumor immune microenvironment is important during tumor progress, we also study the CDK6-AS1 expression with infiltrating immune cells fractions. Intriguingly, we found that lower CDK6-AS1 expression shown positive tendencies with antitumor immune cells proportions such as T helper cells. This could partially

explained by the finding that low CDK6-AS1 expression group had more mutated genes than high expression ones. More frequent mutated genes indicts more mutational burden and more cancer neoantigen(29). Neoantigen could serve as target for immune recognition and immune cells recruitment(30). Relationship between CDK6-AS1 and neoantigen is worthy of further study.

Undeniably, our research had some limitations. Firstly, the sample of this research is small, and more clincial researches are needed. Secondly, the more detail of CDK6-AS1 in gastric cancer are needed to further research.

\section{Conclusion}


CDK6-AS1 may serve as a poor independent prognostic biomarker candidate in gastric cancer with positive correlation with its target gene CDK6. Moreover, CDK6-AS1 is involved in key oncogenic pathway as cell cycle and RNA transcription. CDK6-AS1 also shows dysregulations and associations with prognosis at pan-cancer level. This eRNA may also associated with immune cell infiltration and drug sensitivity.

\section{Declarations}

\section{Ethics approval and consent to participate}

The studies involving human participants were reviewed and approved by the Ethics Committee of the Second Affiliated Hospital of Harbin Medical University. The patients/participants provided their written informed consent to participate in this study.

\section{Consent for publication}

All authors have read and approved the manuscript being submitted囚and agree to its publicated to this journal.

\section{Availability of data and materials}

The datasets presented in this study can be found in online repositories. The names of the repository/repositories and accession number(s) can be found in the article/Supplementary Material.

\section{Competing interests}

There is no conflict of interest in this article.

\section{Funding}

This project was supported by the National Natural Science Foundation of China (81672355).

\section{Author contributions}

SY and XZ designed the study. HY,JL and AZ performed the experiment. LZ, and ZM analyzed the data. SY and QC wrote the manuscript. All authors revi manuscript.

\section{Acknowledgments}

The authors want to thank all the participants in the research.

\section{References}

1. Bray F, Ferlay J, Soerjomataram I, Siegel RL, Torre LA, Jemal A. Global cancer statistics 2018: GLOBOCAN estimates of incidence and mortality worldwide for 36 cancers in 185 countries. Journal ofimmunology (2018) 68(6):394-424. doi: 10.4049/jimmunol. 1800242 10.3322/caac.21492.

2. Thrift AP, El-Serag HB. Burden of Gastric Cancer. Cancers (2020) 18(3):534-42. doi:10.3390/cancers12051062 10. 1016/j.cgh.2019.07.045.

3. Soerjomataram I, Lortet-Tieulent J, Parkin DM, Ferlay J, Mathers C, Forman D, et al. Global burden of cancer in 2008: a systematic analysis of disabilityadjusted life-years in 12 world regions. Lancet (2012) 380(9856):1840-50. doi: 10. 1016/s0140-6736(12)60919-2.

4. Comprehensive molecular characterization of gastric adenocarcinoma. Nature (2014) 513(7517):2029. doi: 10. $1038 /$ nature13480.

5. Digklia A, Wagner AD. Advanced gastric cancer: Current treatment landscape and future perspectives. World journal of gastroenterology (2016) 22(8):2403- 14. doi: 10.3748/wjg.v22.i8.2403.

6. Martens-Uzunova ES, Böttcher R, Croce CM, Jenster G, Visakorpi T, Calin GA. Long noncoding RNA in prostate, bladder, and kidney cancer. European urology (2014) 65(6):1140-51. doi: 10. 1016/j.eururo.2013. 12.003.

7. NatoliG,AndrauJC.Noncodingtranscriptionatenhancers:generalprinciplesandfunctionalmodels.Annual review ofgenetics (2012)46:119.doi:10.1146/annurev-genet-110711-155459.

8. Andersson R, Gebhard C, Miguel-Escalada I, Hoof I, Bornholdt J, Boyd M, et al. An atlas of active enhancers across human cell types and tissues. Nature (2014) 507(7493):455-61. doi: 10. 1038/nature12787

9. Kaikkonen MU, Spann NJ, Heinz S, Romanoski CE, Allison KA, Stender JD, et al. Remodeling of the enhancer landscape during macrophage activation is coupled to enhancer transcription. Molecular cell (2013) 51(3):310-25. doi: 10. 1016/j.molcel.2013.07.010.

10. Kim TK, Shiekhattar R. Architectural and Functional Commonalities between Enhancers and Promoters. Cell (2015) 162(5):948-59. doi: 10. 1016/j.cell.2015.08.008. 
11. Kjems J, Zhang Z. Transcriptional landscape and clinical utility of enhancer RNAs for eRNA-targeted therapy in cancer. Nature reviews Genetics (2019) 10(1):4562. doi:10. 1038/s41576-019-0158-7 10. 1038/s41467-019-12543-5.

12. Hsieh CL, Fei T, Chen Y, Li T, Gao Y, Wang X, et al. Enhancer RNAs participate in androgen receptor-driven looping that selectively enhances gene activation. Proceedings of the National Academy of Sciences of the United States of America (2014) 111(20):7319-24. doi: 10. 1073/pnas. 1324151111.

13. Xiao J, Liu Y, Yi J, Liu X. LINC02257, an Enhancer RNA of Prognostic Value in Colon Adenocarcinoma, Correlates With Multi-Omics ImmunotherapyRelated Analysis in 33 Cancers. Frontiers in molecular biosciences (2021) 8:646786. doi: 10. 1186/s12885-021-08517-w 10.3389/fmolb.2021.646786.

14. Miracco C, Giannicola R, Giordano A, Pirtoli L, Cheng L, Han T, et al. TBX5-AS1, an enhancer RNA, is a potential novel prognostic biomarker for lung adenocarcinoma. International journal ofmolecular sciences (2021) 21(1):794. doi: 10.3390/ijms22168391 10. 1186/s12885-021-08517-w.

15. Gu X, Wang L, Boldrup L, Coates PJ, Fahraeus R, Sgaramella N, et al. AP001056. 1, A Prognosis-Related Enhancer RNA in Squamous Cell Carcinoma of the Head and Neck. (2019) 11(3). doi: 10.3389/fimmu.2019.01392 10.3390/cancers11030347.

16. Goldman MJ, Craft B. Visualizing and interpreting cancer genomics data via the Xena platform. (2020) 38(6):675-8. doi: 10. 1080/15548627.2020. 1713646 10. 1038/s41587-020-0546-8.

17. Corradin O, Saiakhova A, Akhtar-Zaidi B, Myeroff L, Willis J, Cowper-al lari R, et al. Combinatorial effects of multiple enhancer variants in linkage disequilibrium dictate levels of gene expression to confer susceptibility to common traits. Genome research (2014) 24(1):1-13. doi: 10. 1101/gr. 164079. 113.

18. Li B, Severson E, Pignon JC, Zhao H, Li T, Novak J, et al. Comprehensive analyses of tumor immunity: implications for cancer immunotherapy. Genome biology (2016) 17(1):174. doi:10. 1186/s13059-016- 1028-7.

19. Geeleher P, Zhang Z, Wang F, Gruener RF, Nath A, Morrison G, et al. Discovering novel pharmacogenomic biomarkers by imputing drug response in cancer patients from large genomics studies. Nature medicine (2017) 27(10):1743-51. doi: 10. 1038/nm.4368 10. 1101/gr.221077. 117.

20. Bresnick EH, Johnson KD. Blood disease-causing and -suppressing transcriptional enhancers: general principles and GATA2 mechanisms. Nature communications (2019) 3(13):2045-56. doi: 10. 1038/s41467-019- 12543-5 10. 1182/bloodadvances.2019000378.

21. Bal E, Park HS, Belaid-Choucair Z, Kayserili H, Naville M, Madrange M, et al. Mutations in ACTRT1 and its enhancer RNA elements lead to aberrant activation of Hedgehog signaling in inherited and sporadic basal cell carcinomas. (2017) 23(10):1226-33. doi: 10. 1007/s00109-017- 1582-9 10. 1038/nm.4368.

22. Nebenfuehr S, Kollmann K, SexI V. The role of CDK6 in cancer. (2020) 147(11):2988-95. doi: 10.3389/fmolb.2021.646786 10. $1002 / \mathrm{ijc} .33054$.

23. Nardone V, Barbarino M. CDK4, CDK6/cyclin-D1 Complex Inhibition and Radiotherapy for Cancer Control: A Role for Autophagy. (2021) 22(16). doi: 10.3390/ijms22168391.

24. Song Z, Wei Z, Wang Q, Zhang X, Tao X, Wu N, et al. The role of DOT1L in the proliferation and prognosis of gastric cancer. Bioscience reports (2020) 40(1). doi: 10. 1016/j.cgh.2019.07.045 10. 1042/bsr20193515.

25. Malumbres M, Barbacid M. Cell cycle, CDKs and cancer: a changing paradigm. Nature reviews Cancer (2009) 9(3):153-66. doi: 10. 1038/nrc2602.

26. Scheicher R, Hoelbl-Kovacic A, Bellutti F, Tigan AS, Prchal-Murphy M, Heller G, et al. CDK6 as a key regulator of hematopoietic and leukemic stem cell activation. Blood (2015) 125(1):90- 101. doi: 10. 1182/blood-2014-06-584417.

27. Scheicher R, HoelbIKovacic A, Bellutti F, et al. CDK6 as a key regulator of hematopoietic and leukemic stem cell activation. Blood. 2015;125(1):90-101. Blood (2018) 132(9):978-9. doi: 10.3389/fonc.2018.00398 10. 1182/blood-2018-07-863001.

28. Goel S, DeCristo MJ, Watt AC, BrinJones H, Sceneay J, Li BB, et al. CDK4/6 inhibition triggers anti-tumour immunity. Nature (2017) 548(7668):471-5. doi: 10. 1038 /nature23465.

29. Xu P, Luo H, Kong Y, Lai WF, Cui L, Zhu X. Cancer neoantigen: Boosting immunotherapy. Internationaljournal ofcancer (2020) 131:110640. doi: 10. 1002/ijc.33054 10. 1016/j.biopha.2020. 110640.

30. Garcia-Garijo A, Fajardo CA, Gros A. Determinants for Neoantigen Identification. Cell death \& disease (2019) 10:1392. doi: 10. 1038/s41419-019-1744-5 10.3389/fimmu.2019.01392.

\section{Tables}

Due to technical limitations, tables are only available as a download in the Supplemental Files section.

\section{Figures}



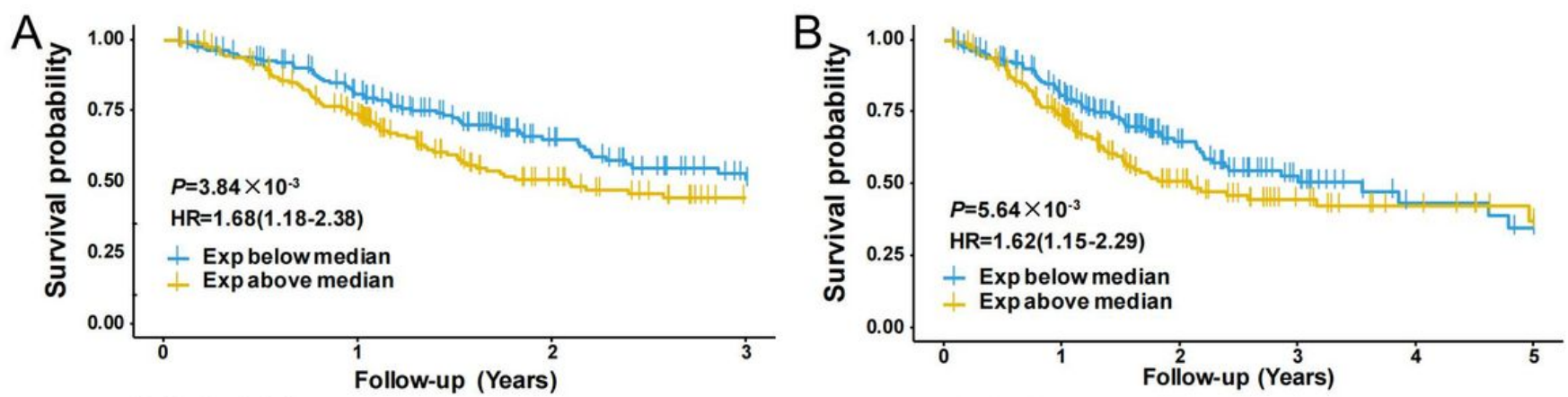

Patients at risk
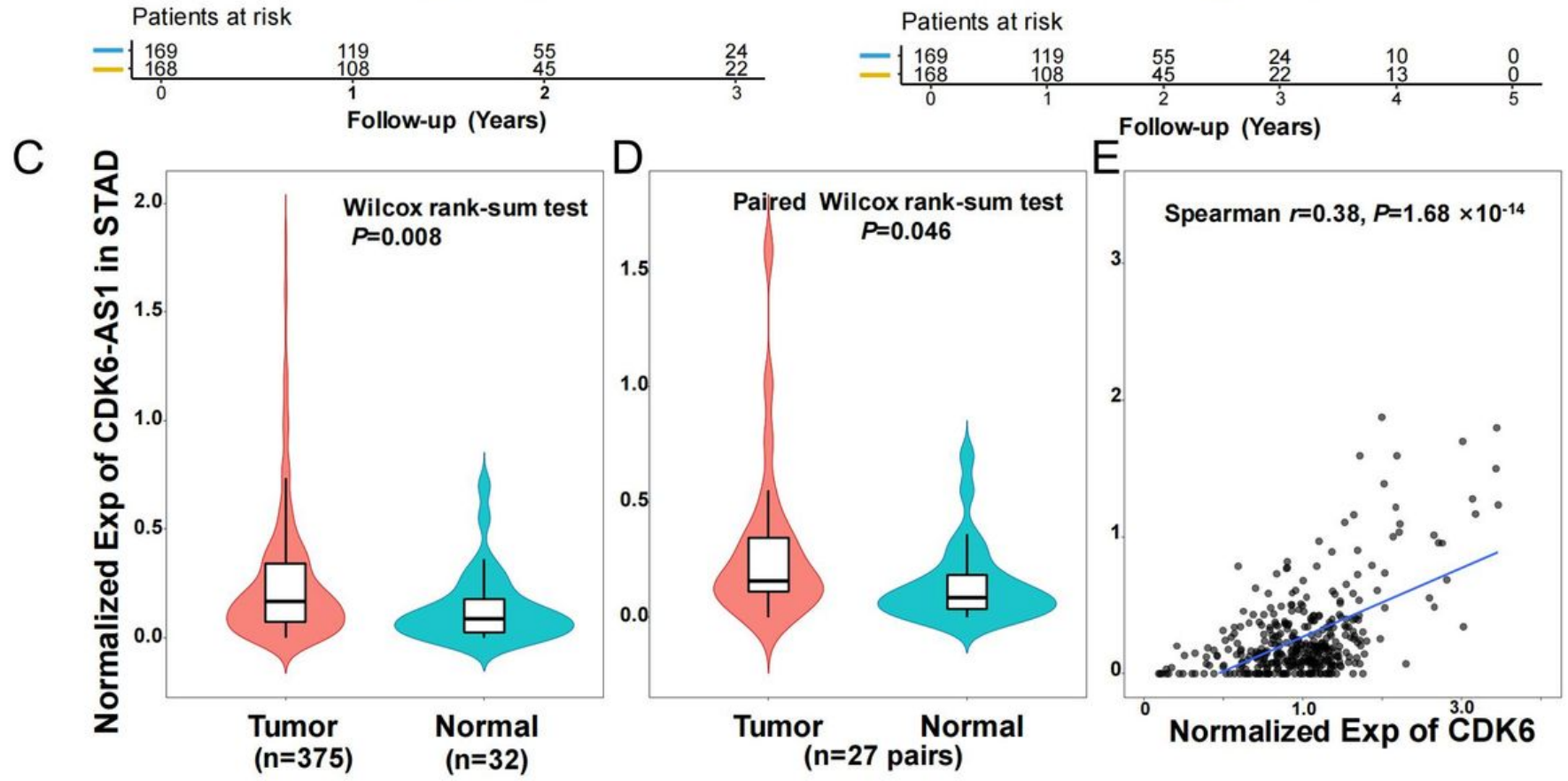

Figure 1

Characteristics of eRNA CDK6-AS1 in gastric cancer .

(A)Kaplan-Meier 3-year overall survival curve for gastric cancer patients with CDK6-AS1 low and high expression.

(B) Kaplan-Meier 5-year overall survival curve for gastric cancer patients with CDK6-AS1 low and high expression.

(C) Differential expression of CDK6-AS1 between unpaired tumor and adjacent normal tissues.

(D)Differential expression of CDK6-AS1 between paired tumor and adjacent normal tissues.

(E) The correlation between the CDK6-AS1 and its target gene CDK6 expression levels. 

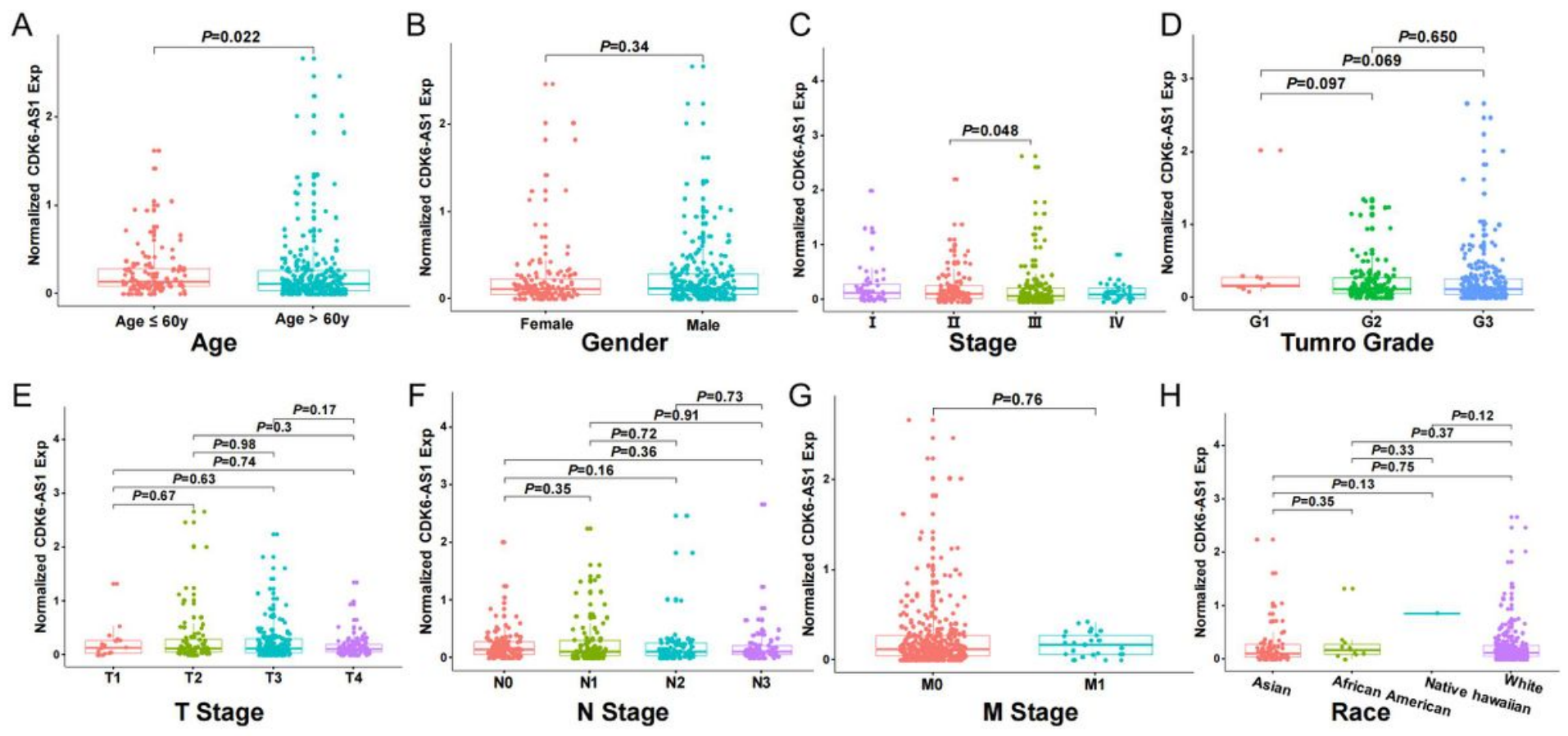

\section{Figure 2}

The relationship between $C D K 6-A S 1$ expression and clinical features.

(A-H) The expression of CDK6-AS1 among patients with age $\geq 60$ years and $<60$ years, male and female, $\varangle-\otimes$ clinical stages, G1-3 grades, T1-4 stages, N0-3 stages, M0- 1 stages, and different races, 


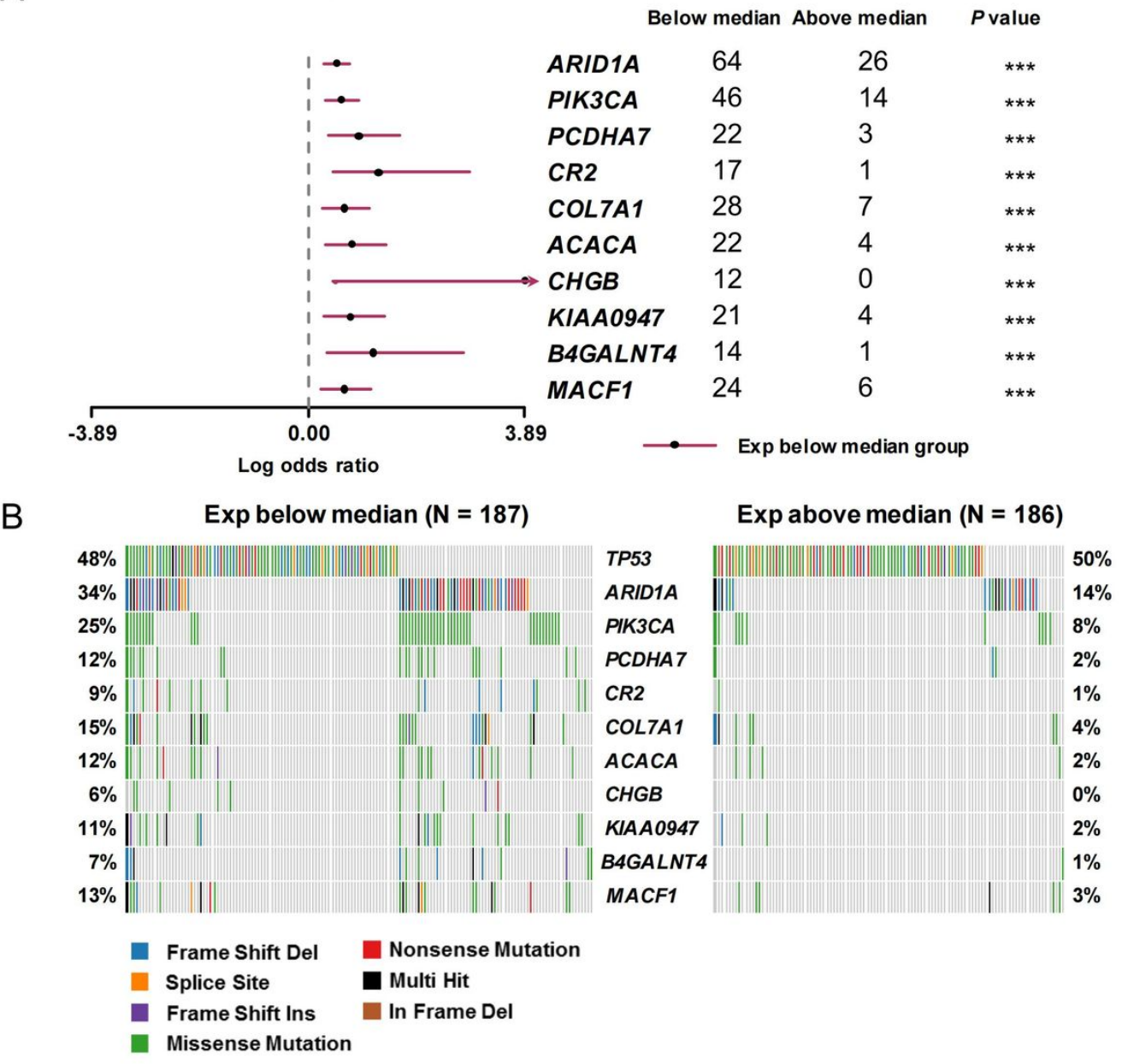

Figure 3

Frequencies of mutated genes between different CDK6-AS1 expression

group

(A)Forest plot showed top frequencies of mutated genes between high and low CDK6-AS1 expression groups.

(B) Waterfall plot side by side for comparison on different mutated genes and mutated types between high and low CDK6-AS1 expression groups. 
A

establishment of RNA localization protein export from nucleus ncRNA export from nucleus

RNA export from nucleus RNA localization RNA transport

nucleic acid transport

TRNA complex transportation tRNA export from nucleus

structural constituent of nuclear pore histone binding catalytic activity, acting on RNA helicase activity 3'-5'-exoribonuclease activity exonuclease activity exoribonuclease activity exoribonuclease activity protein serine/threonine kinase activity exonuclease activity

nuclear membran chromosomal region nuclear pore nuclear envelope host ce

other organism part other organism cell other organism chromosome, centromeric region

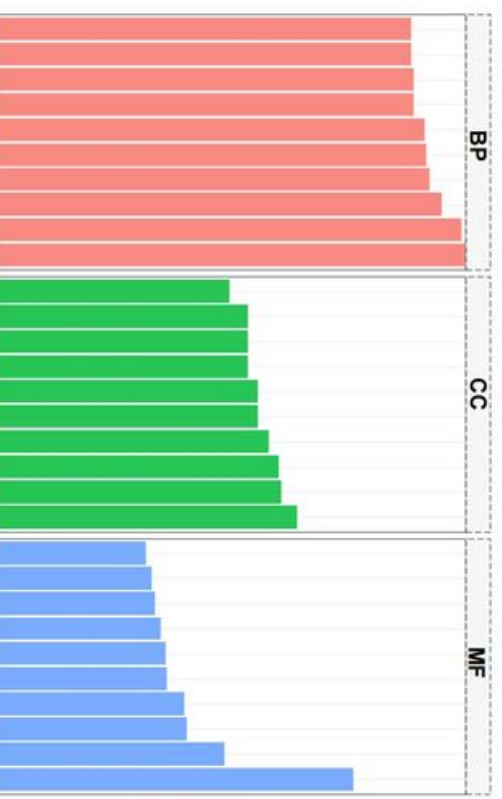

$-\log P$ of enrichment
B

Regulation of HSF1-mediated heat shock response SUMOylation of DNA damage response and repair proteins SUMO E3 ligases SUMOylate target proteins Mitotic Prometaphase Resolution of Sister Chromatid Cohesion Rev-mediated nuclear export of HIV RNA Interactions of Rev with host cellular proteins ISG15 antiviral mechanism Antiviral mechanism by IFN-stimulated genes Transcriptional regulation by small RNAs Mitotic Prophase

Viral Messenger RNA Synthesis Regulation of Glucokinase by Glucokinase Regulatory Protein Nuclear import of Rev protein Vpr-mediated nuclear import of PICs Nuclear Pore Complex (NPC) Disassembly. Interactions of $\mathrm{Vpr}$ with host cellular proteins Glucose transport Hexose transport Nuclear Envelope Breakdown

$$
\begin{array}{lllll}
0.05 & 0.10 & 0.15 & 0.20 & 0.25
\end{array}
$$

Gene Enrichment Ratio

\section{Figure 4}

Pathway enrichment analysis of CDK6-AS1 co-expressed genes

(A)GO (Gene Ontology) enrichment result in Biological process (BP), cellular component (CC), and molecular function (MF) categories.

(B) Reactome enrichment result.

Top 30 items were shown 


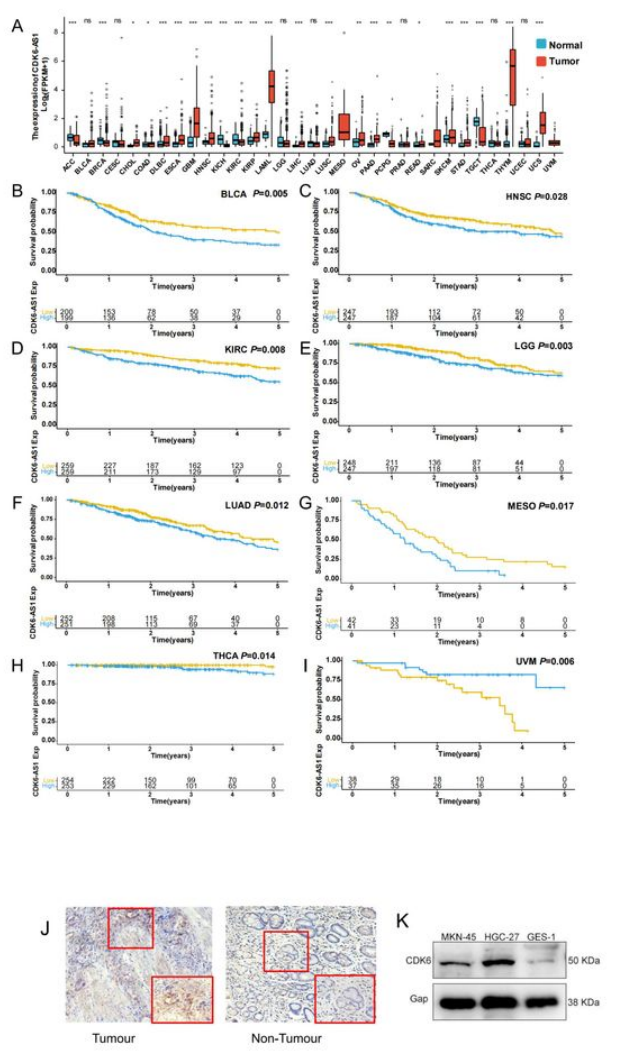

\section{Figure 5}

\section{Expression and survival validation in TCGA pan-cancer cohort.}

(A)Differential expression validation of CDK6-AS1 in TCGA pan-cancer cohort. ${ }^{*} P<0.05 ; * \star P<0.01 ; * \star \star P<0.001$; ns: not significant with $P>0.05$

(B-I) The prognostic effect of CDK6-AS1 in other TCGA cancer types. Types with Cox $P$-value $>0.05$ were shown.

(J)Representative immunohistochemical staining for gastric cance tumor-infiltrating target gene CDK6.

(K)The CDK6 was expressed in gastric cancer cells MKN-45,HGC-27 and gastric mucosa epithelial cells GES- 1. 

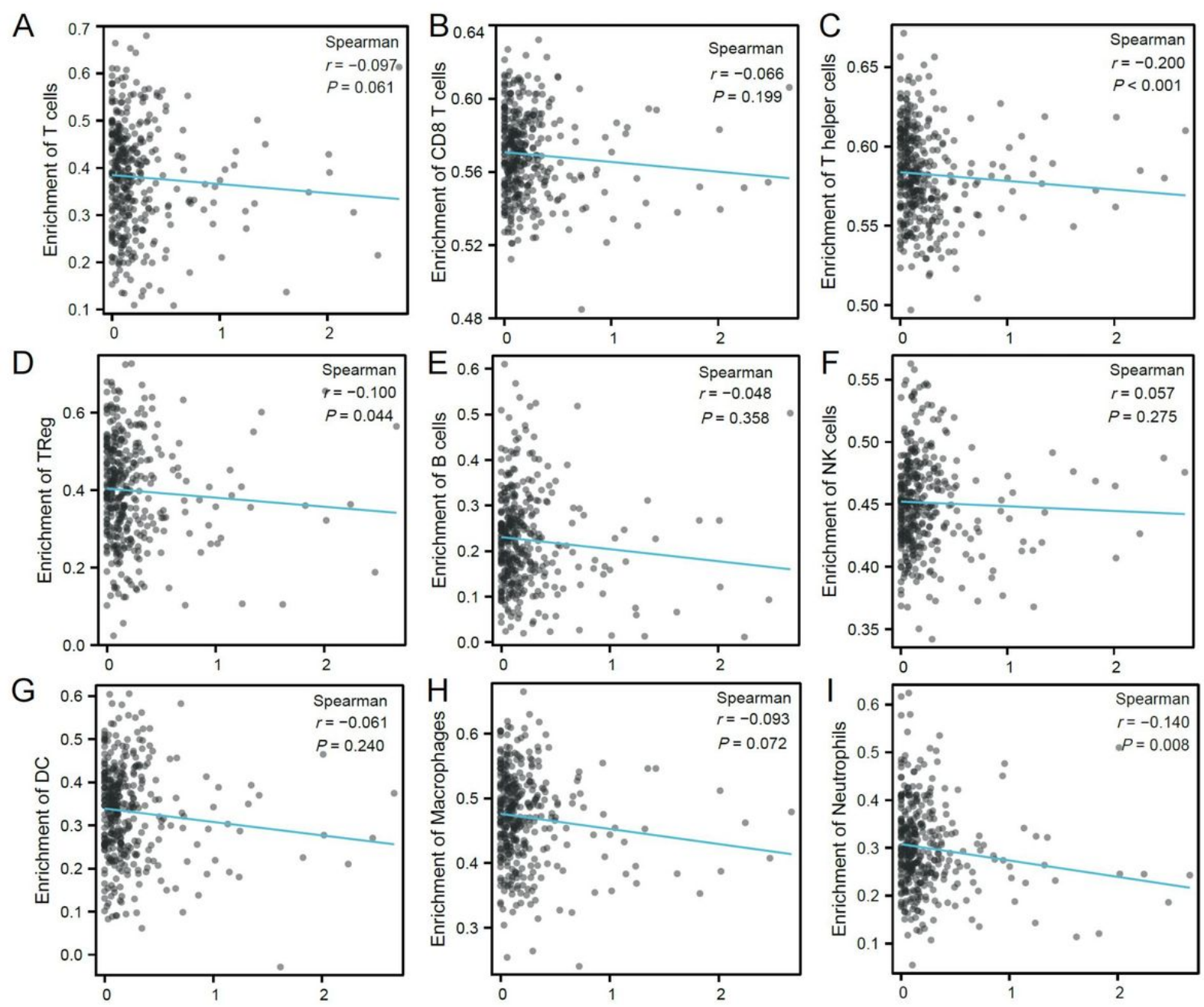

Figure 6

Correlation between expression of CDK6-AS1 and immune cell infiltration proportion.

(A-I) Correlation between expression of CDK6-AS1 and ssGSEA inferred immune cell infiltration en of T, CD8+T, T helper, Treg, B, NK, DC, macrophages, and neutrophils cells. 

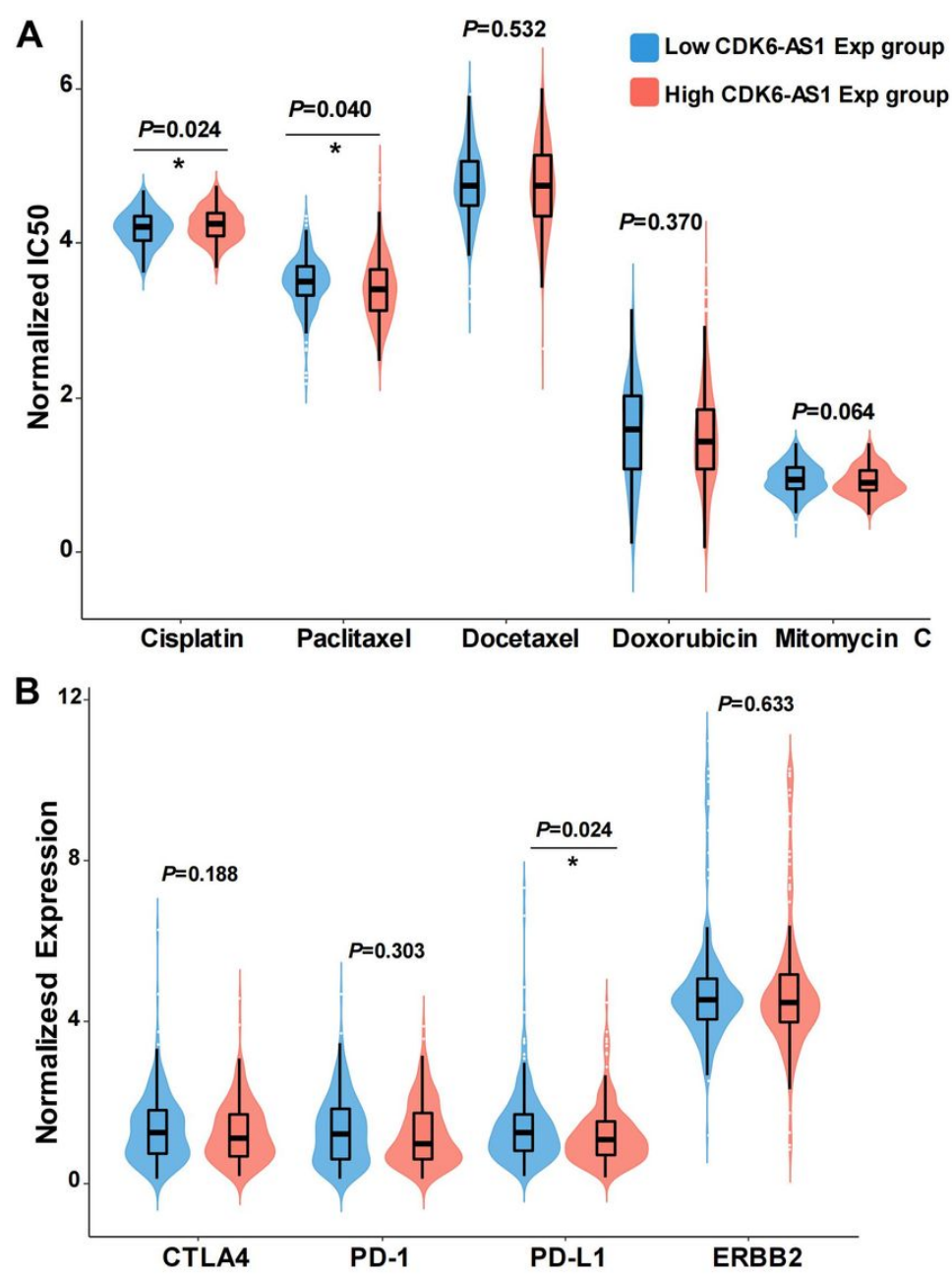

Figure 7

Drug sensitivity prediction in low and high CDK6-AS1 expression

\section{group}

(A)Chemosensitivity prediction of five commonly used drugs in antineoplastic therapy between low and high CDK6-AS1 expression group. IC50:half maximal inhibitory concentration.

(B) Expressions of immunotherapy and HER2-targeted therapy markers between low and high CDK6-AS1 expression group.

\section{Supplementary Files}

This is a list of supplementary files associated with this preprint. Click to download.

- TablesV2.xlsx 